\title{
An Asymmetric, Energetic Type Ic Supernova Viewed Off-Axis, and a Link to Gamma-Ray Bursts
}

\author{
Paolo A. Mazzali ${ }^{1,2,3,4 *}$, Koji S. Kawabata ${ }^{5}$, Keiichi Maeda ${ }^{6}$, Ken'ichi Nomoto $^{1,2 * *}$, \\ Alexei V. Filippenko ${ }^{7}$, Enrico Ramirez-Ruiz ${ }^{8}$, Stefano Benetti ${ }^{9}$, Elena Pian $^{4}$, \\ Jinsong Deng ${ }^{1,2,10}$, Nozomu Tominaga ${ }^{1}$, Youichi Ohyama ${ }^{11,12}$, Masanori Iye ${ }^{1,13,14}$, \\ Ryan J. Foley ${ }^{7}$, Thomas Matheson ${ }^{15}$, Lifan Wang ${ }^{16}$, and Avishay Gal-Yam ${ }^{17}$
}

\author{
${ }^{1}$ Department of Astronomy, School of Science, University of Tokyo, Bunkyo-ku, Tokyo 113-0033, Japan \\ ${ }^{2}$ Research Center for the Early Universe, School of Science, University of Tokyo, Bunkyo-ku, Tokyo 113-0033, Japan \\ ${ }^{3}$ Max-Planck Institut für Astrophysik, Karl-Schwarzschild Str. 1, D-85748 Garching, Germany \\ ${ }^{4}$ Istituto Nazionale di Astrofisica-OATs, Via Tiepolo 11, I-34131 Trieste, Italy \\ ${ }^{5}$ Hiroshima Astrophysical Science Center, Hiroshima University, Hiroshima 739-8526, Japan \\ ${ }^{6}$ Department of Earth Science and Astronomy, College of Arts and Sciences, \\ University of Tokyo,Komaba 3-8-1, Meguro-ku, Tokyo 153-8902, Japan \\ ${ }^{7}$ Department of Astronomy, University of California, Berkeley, CA 94720-3411, USA \\ ${ }^{8}$ Institute for Advanced Study, Einstein Drive, Princeton, NJ 08540, USA; Chandra Fellow \\ ${ }^{9}$ INAF-Osservatorio Astronomico di Padova, vicolo del'Osservatorio 5, 35122 Padova, Italy \\ ${ }^{10}$ National Astronomical Observatories, CAS 20A Datun Road, Chaoyang District, Beijing 100012, China \\ ${ }^{11}$ Department of Infrared Astrophysics, Institute of Space and Astronautical Science, \\ Japan Aerospace Exploration Agency, 3-1-1 Yoshinodai, Sagamihara, Kanagawa, 229-8510, Japan \\ ${ }^{12}$ Subaru Telescope, NAOJ, 650 North A'ohoku Place, Hilo, HI 96720, USA \\ ${ }^{13}$ Optical and IR Astronomy Division, National Astronomical Observatory, Mitaka, Tokyo 181-8588, Japan \\ ${ }^{14}$ Department of Astronomical Science, School of Physical Sciences, \\ Graduate University for Advanced Studies, 2-21-1 Osawa, Mitaka, Tokyo 181-8588, Japan \\ ${ }^{15}$ National Optical Astronomy Observatory, 950 N. Cherry Avenue, Tucson, AZ 85719-4933, USA \\ ${ }^{16}$ Lawrence Berkeley National Laboratory, 50-232, 1 Cyclotron Road, Berkeley, CA 94720, USA \\ ${ }^{17}$ Department of Astronomy, California Institute of Technology, Pasadena, CA 91125, USA; Hubble Fellow \\ *mazzali@ts.astro.it, ${ }^{* *}$ nomoto@astron.s.u-tokyo.ac.jp
}

\section{To be published in Science on 27 May 2005.}


Type Ic supernovae, the explosions following the core collapse of massive stars that have previously lost their hydrogen and helium envelopes, are particularly interesting because of the link with longduration gamma-ray bursts. Although indications exist that these explosions are aspherical, direct evidence has still been missing. Late-time observations of SN 2003jd, a luminous Type Ic supernova, provide such evidence. Recent Subaru and Keck spectra reveal double-peaked profiles in the nebular lines of neutral oxygen and magnesium. These profiles are different from those of known Type Ic supernovae, with or without a gamma-ray burst, and they can be understood if SN 2003jd was an aspherical, axisymmetric explosion viewed from near the equatorial plane. If SN 2003jd was associated with a gamma-ray burst, we missed the burst as it was pointing away from us.

When a massive star reaches the end of its life and exhausts its nuclear fuel, the core itself collapses to form a compact remnant (a neutron star or a black hole). Although the exact mechanism is not well-understood, the resulting release of energy leads to the ejection of the envelope of the star at high velocities, producing a supernova (SN).

Typically, a massive star has a large H-rich envelope, making it difficult to observe the innermost part, where the action takes place. There are, however, some cases where the $\mathrm{H}$ envelope, and also the inner He envelope, were lost before the star exploded, through either a stellar wind or, more likely, binary interaction (1). These SNe, called Type Ic, offer a view close to the core, and so they are particularly interesting as tools to study the properties of the collapse and of the SN ejection.

Adding to this, some SNe Ic, characterized by a very high kinetic energy (2,3), have 
been observed to be linked with the previously unexplained phenomenon of gamma-ray bursts (GRBs) - brief but extremely bright flashes of hard ( $\gamma$-ray and X-ray) radiation which for decades had baffled astronomers $(4-8)$. The link between SNe Ic and GRBs is probably not accidental. If a jet is produced by a collapsing star, it can only emerge and generate a GRB if the stellar envelope does not interfere with it (9).

In view of this link, we have searched among known SNe Ic for the counterpart of a property that is typical of GRBs: asphericity. A jet-like explosion is required for GRBs from energetics considerations: if they were spherically symmetric, GRBs would involve excessively large energies, comparable to the rest mass of several suns. The best evidence for asphericity in the GRB-associated $\mathrm{SNe}(\mathrm{GRB} / \mathrm{SNe})$ has so far come from the fact that iron seems to move faster than oxygen in the ejected material. Evidence of this is seen in spectra obtained several months after the explosion, when the ejected material has decreased in density and behaves like a nebula. The GRB/SN 1998bw (10) showed strong emission lines of [O I] (a forbidden line of neutral oxygen), as do normal SNe Ic, but also of [Fe II] (a forbidden line of singly-ionized iron), which are weak in normal SNe Ic. The [Fe II] lines near $5100 \AA$ in SN 1998bw are broader than the [O I] 6300, $6363 \AA$ blend.

Asphericity can explain this peculiar situation (11). In a typical, spherical SN explosion, heavier elements are produced in deeper layers of the progenitor star, and as a consequence of the hydrodynamical properties of the explosion they are given less kinetic energy per unit mass than external layers, which typically contain lighter elements. However, in a jet-like explosion the heavier elements (in particular ${ }^{56} \mathrm{Ni}$ ) are probably synthesized near the jet at the time of core collapse, and are ejected at high velocities. Lighter elements such as oxygen, which are not produced in the explosion but rather by the progenitor star during its evolution, are ejected near the equatorial plane with a smaller kinetic energy, and are distributed in a disc-like structure. 
Given this scenario, the observed line profiles depend on the orientation of the explosion with respect to our line of sight. Iron can be observed to be approaching us at a higher velocity than oxygen if we view the explosion near the jet direction, which is also the requirement for the GRB to be observed $(10,11)$. The [O I] line, on the other hand, will appear as a narrow, sharp line in the case of a polar view, since in that case oxygen moves almost perpendicular with respect to our line of sight (the case of SN 1998bw), but it will show a broader, double-peaked profile for an equatorial view, since a large fraction of the oxygen would then be moving either toward or away from the observer (11).

While this picture seems well established for GRB/SNe, it is important to determine whether it may be common to other SNe Ic. Measurements of the relative widths of the Fe and $\mathrm{O}$ lines are difficult for fainter SNe Ic because the Fe lines are weak. However, the [O I] line is always rather strong, and it can be expected that, given a sufficiently large sample, variations should be seen in its profile reflecting different viewing angles.

So far the evidence for this was missing (12), but recent observations of SN 2003jd seem to close this gap. SN 2003jd, discovered on 25 Oct. 2003 (13) (UT dates are used in this paper), is a SN Ic at a distance of $\sim 80 \mathrm{Mpc}$ and reached a rather bright maximum. Assuming a Galactic extinction $E(B-V)_{\text {Gal }}=0.06 \mathrm{mag}$ (14), and a host extinction $E(B-V)_{\text {Host }}=0.09 \mathrm{mag}$, as derived from the strength of the interstellar Na I D absorption $(15)$, we derive $M_{B}(\operatorname{Max}) \approx-18.7 \mathrm{mag}$. This is much more luminous than the normal SN Ic 1994I (1,16), and is comparable to the GRB/SNe 1998bw (4) and 2003dh (6). Spectroscopically, however, SN 2003jd shows narrower lines than the hyper-energetic GRB/SNe (Fig. 1), and it appears to be intermediate between those and the normal SN Ic 1994I (16). The closest analogue may be the energetic SN 2002ap $(17,18)$.

We observed SN 2003jd in the nebular phase with the Japanese 8.2-m Subaru telescope on 12 Sep. 2004 (19) using the FOCAS spectrograph (20), and with the 10-m Keck-I 
telescope on 19 Oct. 2004 using LRIS (21). These dates correspond to SN ages of 330 and $\sim 370$ days after explosion, respectively. In both spectra (Fig. 2) the nebular line [O I] $6300,6363 \AA$ clearly has a double-peaked profile with FWHM $\approx 8000 \mathrm{~km} \mathrm{~s}^{-1}$. The Mg I] $4570 \AA$ line shows a similar profile. Magnesium is formed near oxygen in the progenitor star. The $[\mathrm{Fe}$ II] blend near $5100 \AA$ is quite weak.

Late-phase emission is created by the release of the heat deposited by the $\gamma$-rays and the positrons emitted in the decay chain ${ }^{56} \mathrm{Ni} \rightarrow{ }^{56} \mathrm{Co} \rightarrow{ }^{56} \mathrm{Fe}$. Therefore, the mass of ${ }^{56} \mathrm{Ni}$ can be determined indirectly through the strength of the emission lines. Since SN 2003jd was not as luminous as SN 1998bw, we rescaled the synthetic spectra to the appropriate ${ }^{56} \mathrm{Ni}$ mass, the best value for which was $\sim 0.3 M_{\odot}$. This is actually very similar to that derived for the GRB-associated SN 2003dh (22,23), and much larger than in the non-GRB SNe Ic (e.g., 17).

We computed nebular spectra of 2D explosion models for various asphericities and orientations (11). We found that a spherical model produces a flat-topped [O I] profile, which is not compatible with either SN 1998bw or SN 2003jd. The flat-topped emission is a typical characteristic of emission from a shell. Indeed, the emission from any spherically distributing materials should have the maximum at the wavelength of the line transition between $6300-6363 \AA$, taking into account the line blending. On the other hand, Fig. 3 shows that a highly aspherical model can explain the [O I] line profiles in both SN 1998bw and SN 2003jd. To reproduce the double-peaked profile of the [O I] line in SN 2003jd we found that SN 2003jd must be oriented $\gtrsim 70^{\circ}$ away from our line of sight. In contrast, for SN 1998bw this angle was only $\sim 15-30^{\circ}$ (11), and it was even smaller for SN 2003dh (24). Less aspherical models do not produce sufficiently sharp [O I] in SN 1998bw.

This result confirms that SN 2003jd is a significantly aspherical explosion, and raises the interesting question of whether SN 2003jd was itself a GRB/SN. A GRB was not 
detected in coincidence with SN 2003jd (25).

If the explosion was very off-axis, we do not anticipate to have been able to detect $\gamma$-rays. However, a GRB is expected to produce a long-lived radiative output through synchrotron emission. X-ray and radio emission are produced by the deceleration of the relativistic jet as it expands into the wind emitted by the progenitor star before it exploded. This afterglow emission is very weak until the Doppler cone of the beam intersects our line of sight, making off-axis GRB jets directly detectable only months after the event, and at long wavelengths.

SN 2003jd was observed in X-rays with Chandra on 10 Nov. 2004, about 30 days after the explosion, and was not detected to a limit $L_{X} \leq 3.8 \times 10^{38} \mathrm{erg} \mathrm{s}^{-1}$ in the energy interval $0.3-2 \mathrm{keV}(26)$. It was also observed in the radio $(8.4 \mathrm{GHz}) 9$ days after the explosion, and again not detected to a limit $L_{R} \leq 10^{27} \mathrm{erg} \mathrm{s}^{-1} \mathrm{~Hz}^{-1}$ (27).

These non-detections may suggest that SN 2003jd did not produce a GRB. However, absence of evidence is not necessarily evidence of absence. Let us consider the standard jet associated with typical GRBs, i.e., a sharp-edged, uniform jet with $E=10^{51} \mathrm{erg}$ and a $5^{\circ}$ opening angle, expanding laterally at the local sound speed (28, 29; see Fig.4 legend for other parameters). If the jet expands in a wind with density $\dot{M} / v=5 \times 10^{11} \mathrm{~g} \mathrm{~cm}^{-1}$ (e.g., mass-loss rate $\dot{M} \sim 10^{-6} M_{\odot} \mathrm{yr}^{-1}$, velocity $v \sim 2000 \mathrm{~km} \mathrm{~s}^{-1}$ ), it would give rise to the X-ray and radio light curves shown in Fig. 4. If the SN made a large angle $\left(\geq 60^{\circ}\right)$ with respect to our line of sight, its associated GRB jet, if present, would not have been detected in the X-rays or radio. Furthermore, the afterglow emission can be fainter for a lower jet energy and/or a lower wind density.

While this does not by itself prove that SN 2003jd produced a GRB, it is certainly a possibility, since quantities such as the ejected mass of ${ }^{56} \mathrm{Ni}$ are comparable to those typical of GRB/SNe (23). Moreover, our observations confirm that the energetic SN 
2003jd is an aspherical explosion, reinforcing the case for a link with a GRB. It could also be the case for other energetic SNe Ic. Note that the lack of X-ray and radio emission does not place stringent constraints on the intrinsic kinetic energy carried by the SN as long as the ejecta experience little deceleration before $\sim 30$ days. The expansion velocity (which need not be isotropic) must be $\lesssim 0.2 A_{*}^{-1 / 3}\left(\epsilon_{e} / 0.1\right)^{-1 / 3} c$ (where $\epsilon_{e}$ is the fraction of the total blast energy that goes into shock-accelerated electrons) in order to produce $L_{X}(t \approx 30$ days $) \leq 3.8 \times 10^{38} \mathrm{erg} \mathrm{s}^{-1}$ for a standard $10^{51} \mathrm{erg}$ SN shell expanding into a wind with density $A_{*}=(\dot{M} / v) /\left(5 \times 10^{11} \mathrm{~g} \mathrm{~cm}^{-1}\right)=1$ (28). The average expansion velocity along our line of sight can be estimated from the early-time spectra; for SN 2003jd this is about $0.05 c$. In an aspherical explosion, however, the kinetic energy must be considerably larger near the rotation axis of the stellar progenitor, with bulk expansion velocities close to $\sim 0.1 c$.

The fact that the bright SN 2003jd is the first SN Ic showing double peaks in the [O I] line (12) suggests that the degree of asphericity is not the same in all SNe Ic. The GRB/SNe (1998bw, 2003dh), which are probably highly aspherical, have been discovered thanks to a GRB trigger; their orientation is therefore such that the [O I] profile must be single-peaked. For normal SNe Ic, which are on average closer and easier to discover, the lack of observed double-peaked profiles suggests that they are not as strongly aspherical. SN 2003jd appears to share many of the properties (energetics, luminosity) of the GRB/SNe, but it was discovered independent of a GRB, and it is likely to be an aspherical SN viewed off-axis. There have been three energetic SNe Ic without a GRB trigger (therefore less biased) — SNe 1997dq, 1997ef, and 2002ap — whose nebular spectra did not show the double peaks in the [O I] $6300 \AA$. Given the small sample, the number (one out of four) is not inconsistent with our interpretation that the viewing angle $\gtrsim 70^{\circ}$ results in the double-peaked [O I]. 
Additional sensitive radio and X-ray observations of SN 2003jd are strongly encouraged, because a jet with the standard parameters could be still detectable by deep observations (Fig. 4). The result should provide valuable tests for the presence of an off-axis GRB with the typical parameters and would further constrain the viewing angle and the mass-loss rate.

\section{References and Notes}

1. K. Nomoto et al., Nature 371, 227 (1994).

2. K. Iwamoto et al., Nature 395, 672 (1998).

3. K. Nomoto et al., Stellar Collapse, ed. C. L. Fryer (Dordrecht: Kluwer), 277 (2004).

4. T. J. Galama et al., Nature 395, 670 (1998).

5. C. Stanek et al., Astrophys. J. 591, L17 (2003).

6. T. Matheson et al., Astrophys. J. 599, 394 (2003).

7. J. Hjorth et al., Nature 423, 847 (2003).

8. J. Malesani et al., Astrophys. J. 609, L5 (2004).

9. A.E. MacFadyen, S.E. Woosley Astrophys. J. 524, 262 (1999).

10. P.A. Mazzali et al., Astrophys. J. 559, 1047 (2001).

11. K. Maeda et al., Astrophys. J. 565, 405 (2002).

12. T. Matheson et al., Astron. J. 121, 1648 (2001).

13. J. Burket, B. Swift, W. Li IAUC 8232 (2003). 
14. D. Schlegel, D. Finkbeiner, M. Davis Astrophys. J. 500, 525 (1998).

15. S. Benetti et al., in preparation.

16. A.V. Filippenko et al., Astrophys. J. 450, L11 (1995).

17. P.A. Mazzali et al., Astrophys. J. 572, L61 (2002).

18. R.J. Foley et al., Publ. Astron. Soc. Pacific 115, 1220 (2003).

19. K. Kawabata et al., IAU Circ. 8410 (2004).

20. N. Kashikawa et al., Publ. Astron. Soc. Japan 54, 819 (2002).

21. J.B. Oke et al., Publ. Astron. Soc. Pacific 107, 375 (1995).

22. J. Deng, N. Tominaga, P.A. Mazzali, K. Maeda, K. Nomoto Astrophys. J. 624, 898 $(2005)$

23. P.A. Mazzali et al., Astrophys. J. 599, L95 (2003).

24. E. Ramirez-Ruiz et al., in preparation.

25. K. Hurley et al., GCN 2439 (2003).

26. D. Watson, E. Pian, J.N. Reeves, J. Hjorth, K. Pedersen GCN 2445 (2003).

27. A.M. Soderberg, S.R. Kulkarni, D.A. Frail GCN 2435 (2003).

28. E. Ramirez-Ruiz, P. Madau Astrophys. J. 608, L89 (2004).

29. J. Granot, E. Ramirez-Ruiz Astrophys. J. 609, L9 (2004).

30. F. Patat et al., Astrophys. J. 555, 900 (2001). 
31. This work is based on data collected at the Subaru telescope operated by NAOJ, and at the Keck telescopes made possible by the W. M. Keck Foundation. This research is supported in part by JSPS and MEXT in Japan, and by NSF in the USA. 


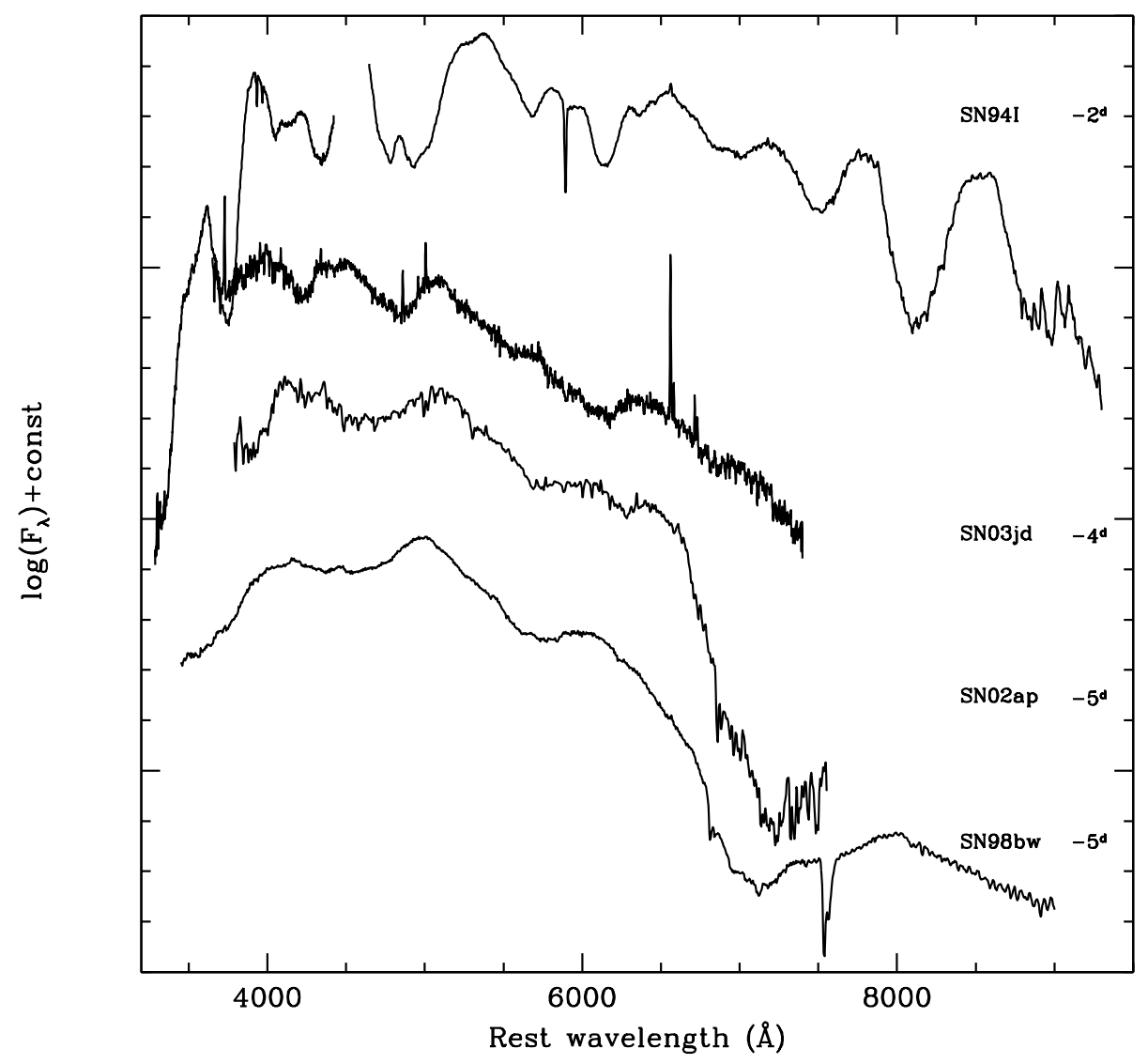

Figure 1: The near-maximum optical spectrum of SN 2003jd compared with spectra of other SNe Ic at a similar phase ( $F_{\lambda}$ is the flux per unit wavelength). The dates are the days relative to the optical maximum (i.e., the minus sign means before the maximum light). Spectra are ordered by increasing line width (implying increasing kinetic energy per unit mass), ranging from the normal SN 1994I (16), to the energetic SN 2002ap (17,18), and to the hyper-energetic GRB/SN 1998bw (30). The absorption line near $7600 \AA$ in the spectrum of SN 1998bw is telluric. 


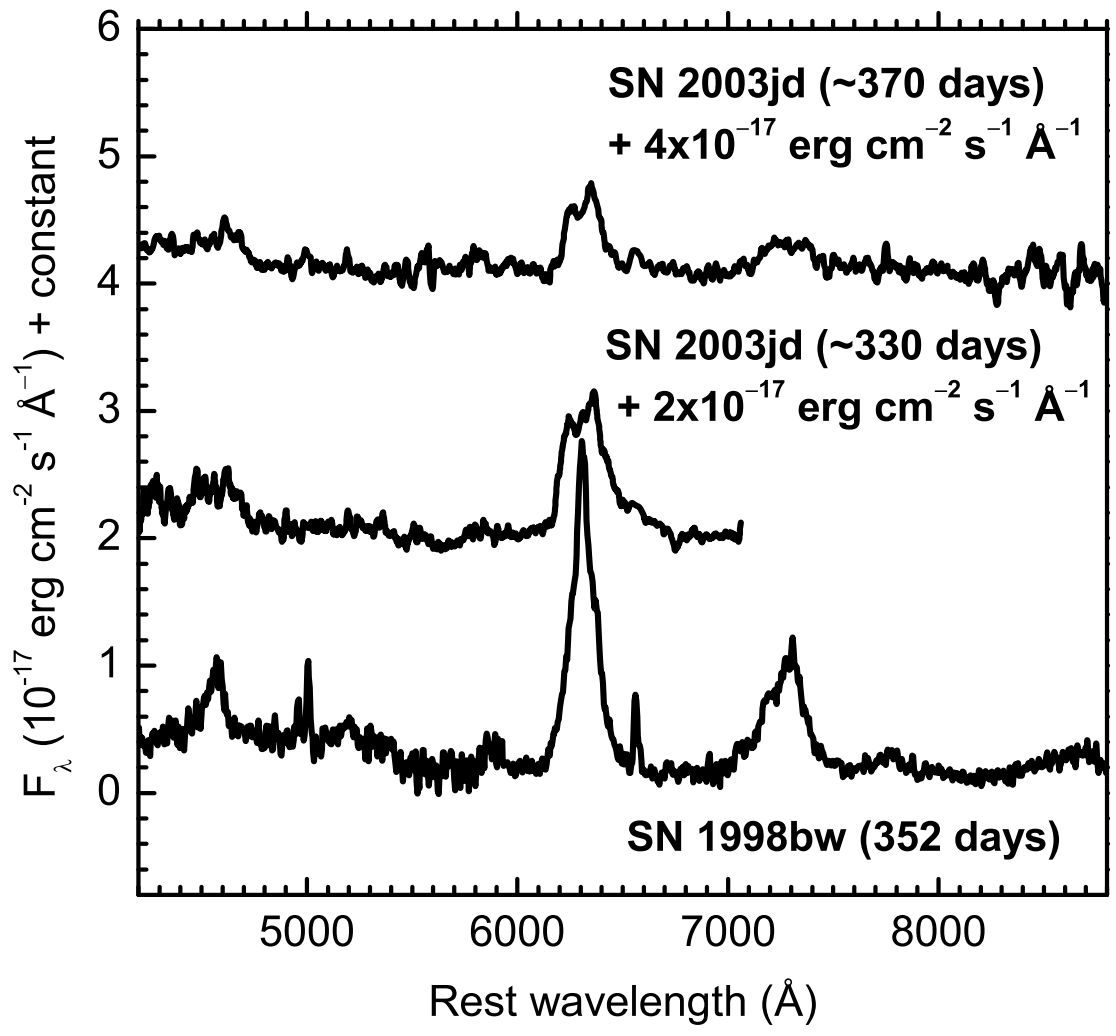

Figure 2: Nebular spectra of SNe Ic. Bottom: nebular spectrum of SN 1998bw (30) taken 337 days after maximum light (352 days after the explosion). Notice the $\mathrm{Mg} \mathrm{I}]$, [Fe II], [O I], and [Ca II] lines near 4570, 5100, 6300, and $7300 \AA$, respectively. Middle: Subaru+FOCAS spectrum of SN 2003jd, 330 days after the putative time of explosion. Top: Keck spectrum of SN 2003jd at an epoch of $\sim 370$ days. The [O I] 6300, $6363 \AA$ line in SN 2003jd clearly exhibits a double-peaked profile. Marginal evidence of a double peak is also present in the profiles of Mg I] $4570 \AA$ and [Ca II] $7300 \AA$. The spectrum of SN 1998bw has been shifted in flux to make it consistent with the distance of SN 2003jd. 

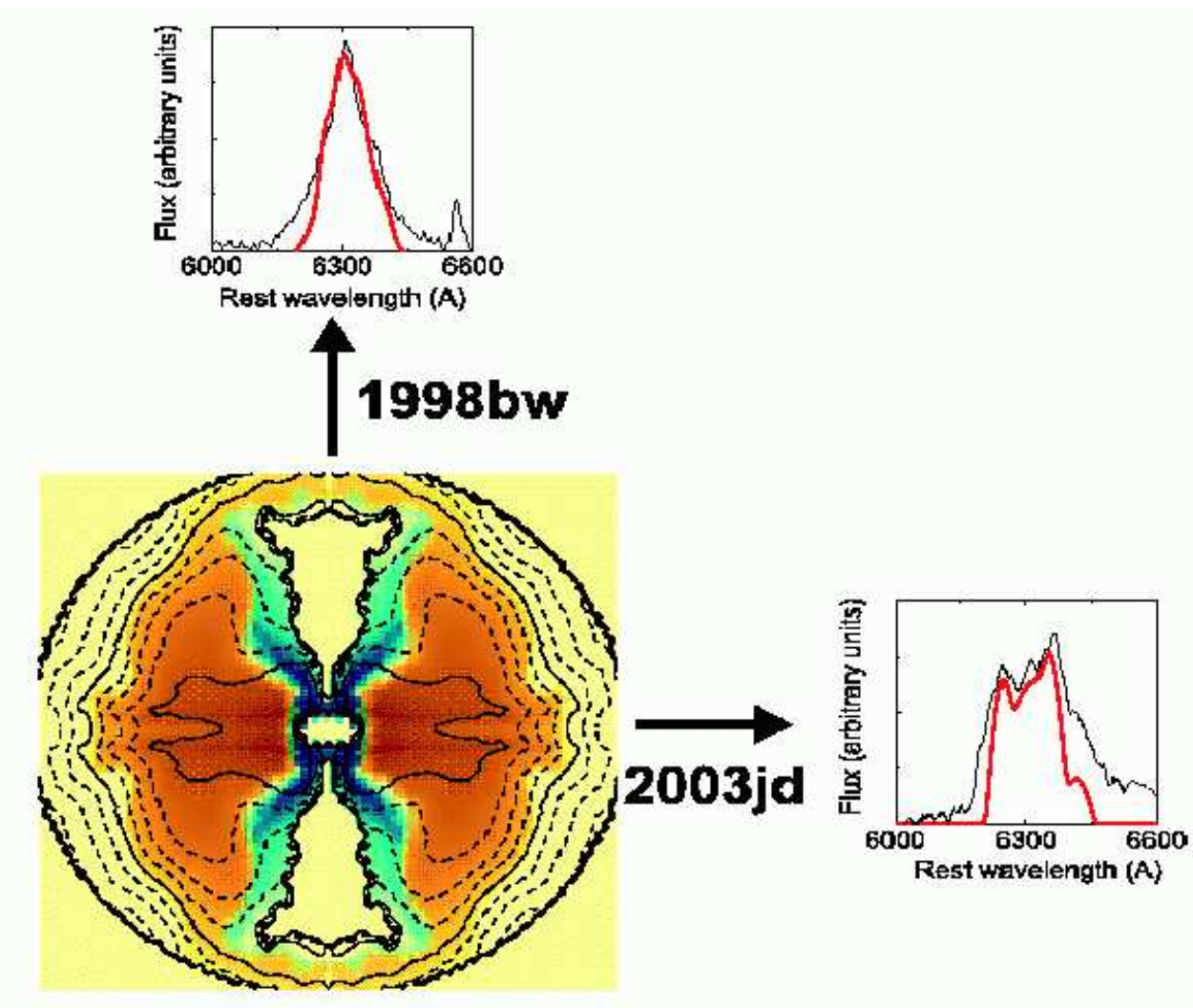

Figure 3: Nebular line profiles observed from an aspherical explosion model depend on the orientation. The figure shows the properties of the explosion model computed in 2D (11): Fe (colored in blue) is ejected near the jet direction and oxygen (brown) in a disc-like structure on and near the equatorial plane. Density contours (covering 2 orders of magnitude and divided into 10 equal intervals in log scale) reflect the dense disc-like structure. Synthetic [O I] 6300, $6363 \AA$ lines (red lines) computed in 2D are compared with the spectra of SN 1998bw and SN 2003jd (black lines). 


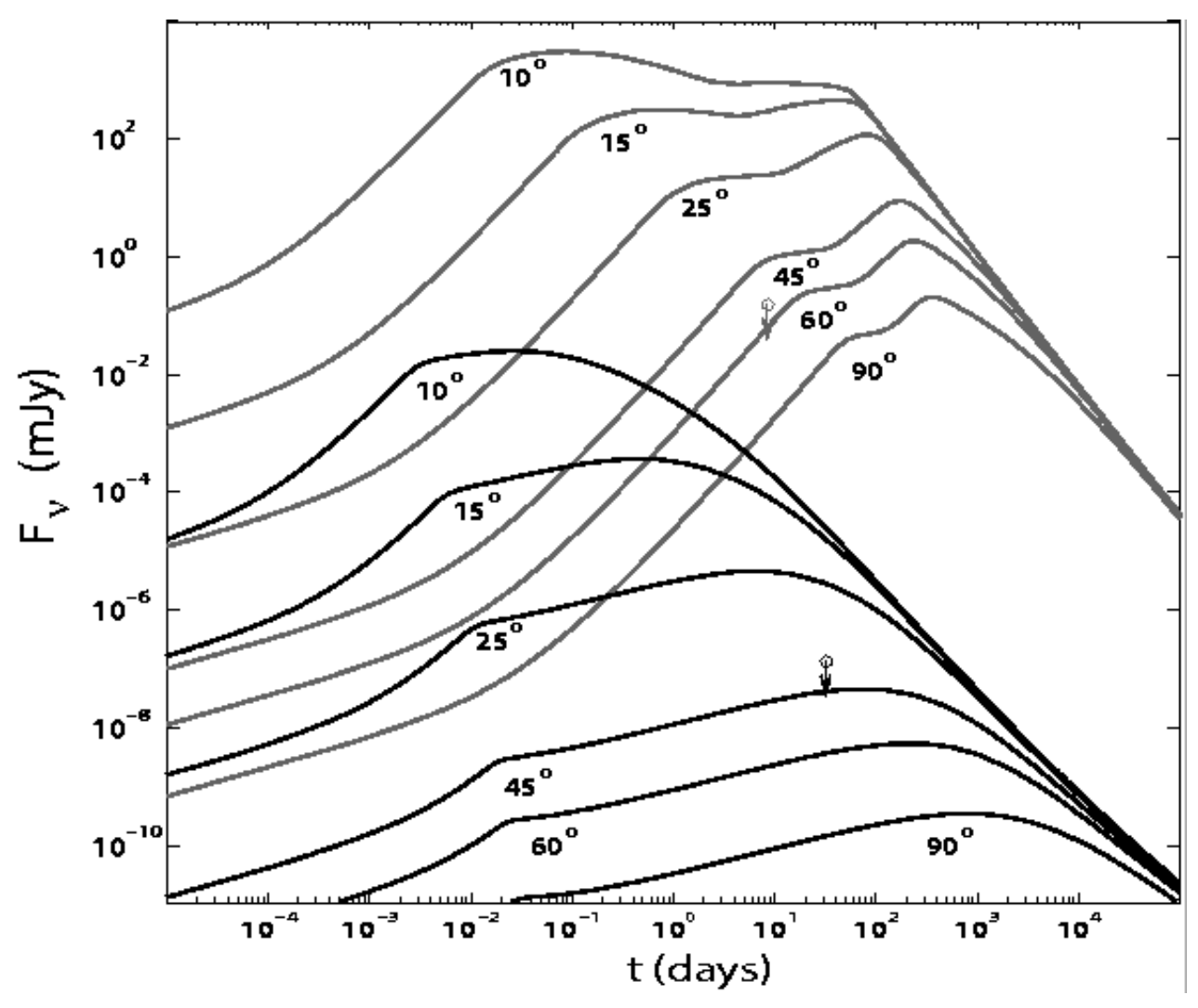

Figure 4: Afterglow emission from a sharp-edged, uniform jet in SN 2003jd. X-ray (0.3$2 \mathrm{keV}$, black) and radio $(8.4 \mathrm{GHz}$, gray) light curves are calculated for various viewing angles $\theta_{\text {obs }}$ for a GRB with the standard parameters $E_{\text {jet }}=10^{51} \mathrm{erg}, \epsilon_{e}=0.1, \epsilon_{B}=0.1$, $\theta_{0}=5^{\circ}$, and $A_{*}=1$ (where $E_{\text {jet }}$ is the energy in the jet, $\epsilon_{e}$ and $\epsilon_{B}$ are the fraction of the internal energy in the electrons and magnetic field, respectively, and $\theta_{0}$ is the opening halfangle of the jet). The synchrotron spectrum is taken to be a piecewise power law with the usual self-absorption, cooling, and injection frequencies calculated from the cooled electron distribution and magnetic field $(28,29)$. The observed radio and X-ray upper limits for SN 2003jd are marked by open circles. Cosmological parameters taken in the model are $\Omega_{\mathrm{m}}=0.27, \Omega_{\Lambda}=0.73$, and $H_{0}=72 \mathrm{~km} \mathrm{~s}^{-1} \mathrm{Mpc}^{-1}$. 